Check for updates

Cite this: RSC Adv., 2018, 8, 12146

Received 10th December 2017 Accepted 17th March 2018

DOI: 10.1039/c7ra13196h

rsc.li/rsc-advances

\title{
Scalable and cost-effective Ag nanowires flexible transparent electrodes
}

\author{
W. W. He, (D) ab X. H. Yan, (D) ${ }^{a}$ Y. M. Liang, (D) a Y. F. Long, (D) ${ }^{a}$ C. Pan, (ID ${ }^{a}$ J. L. Zhao, (D) \\ L. Chen, $\mathbb{D}^{a}$ W. Xiong $\mathbb{D}^{* c}$ and Q. X. Liu (D) ${ }^{* d}$
}

Flexible transparent electrodes (TEs) are important for new electronic devices. This paper reports a scalable, cost effective Ag nanowires (AgNWs) TE, which is made of a $\mathrm{SnO}_{2} \cdot \mathrm{xH}_{2} \mathrm{O}$ and $\mathrm{AgNWs}$ composite layer and a flexible polyethylene terephthalate (PET) bottom layer by a solution method at room temperature. The $\mathrm{AgNWs} / \mathrm{SnO}_{2} \cdot \mathrm{HH}_{2} \mathrm{O}$ composite TEs reveal a significant reduction of four orders in magnitude of sheet

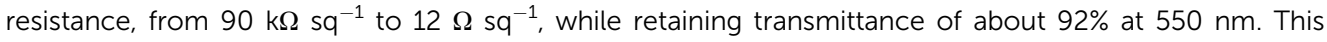
could be owing to the significant reduction of contact resistance for the weld-like junction of bound AgNWs. Compared with others, this method is characterized by filling gaps of the silver nanowire network with $\mathrm{SnO}_{2} \cdot x \mathrm{H}_{2} \mathrm{O}$. In addition, the adhesive forces between the AgNWs and the substrate are improved. This could be attributed to strong adhesion of $\mathrm{SnO}_{2} \cdot \mathrm{xH}_{2} \mathrm{O}$ with the substrate. Moreover, this foldable transparent electrode is applicable for any non-planar surfaces and ultimately for future wearable optoelectronic devices.

\section{Introduction}

A transparent electrode (TE) is an essential component for a wide variety of electronic devices, such as liquid crystal displays, touch screens, organic light emitting diodes (OLEDs), organic photovoltaics (OPVs), microchips, transparent heaters, and smart windows, etc. ${ }^{1-7}$ For electronic devices, indium tin oxide (ITO) with a market share of $93 \%$ is the most widely used TE. ${ }^{8}$ However, further application and development of ITO are expected to be receded due to three issues: scarcity of supply, expensive, and fragile ceramic nature. For these reasons, search for alternatives to ITO is motivated. Obviously, the ultimate market opportunity for alternative TEs is in fully flexible devices. To compete with ITO, the material for the alternatives should possess certain characteristics, for instance, outstanding performance (e.g. transmittance $>95 \%$ and sheet resistance $<100 \Omega \mathrm{sq}^{-1}$ ), flexible, good thermal-cycling, chemically stability, solution-phase coating processes, and costeffective. Recently, carbon based TEs are the most promising alternatives, but still have problems of conductivity, mechanical properties and cost. In addition, metal TEs are also a candidate of the best alternatives. Metal nanowires electrodes can meet

${ }^{a}$ City College, Wuhan University of Science and Technology, Wuhan, Hubei, 430083, China

${ }^{b}$ State Key Laboratory of Materials Processing and Die \& Mould Technology, Huazhong University of Science and Technology, Wuhan 430074, China

'Wuhan National Laboratory for Optoelectronics, Huazhong University of Science and Technology, Wuhan 430074, China. E-mail: weixiong@hust.edu.cn

${ }^{d}$ Urban Construction College, Wuhan University of Science and Technology, Wuhan, Hubei, 430081, China. E-mail: liuqiuxin1956@sohu.com the requirements for the alternatives. The $\mathrm{Ag}$ nanowires (AgNWs) TE has performance even better than ITO, ${ }^{9}$ and is acknowledged as the proverbial "low-hanging fruit" for potential alternative to ITO.

However, pure AgNWs TEs also have three serious problems: low electrical conductivity, intrinsic nonconductive gaps which are not conductive and detrimental to the collection of carriers for the device, and poor adhesion to the substrate.

Firstly, pure AgNWs TEs still have electrical problems because of low contact between the AgNWs. As shown by experimental results, contact resistance of AgNWs $\left(R_{\mathrm{c}}\right)$ is the main source of sheet resistance $\left(R_{\mathrm{sh}}\right)$. The traditional methods for reducing $R_{\mathrm{c}}$ and improving conductivity are heating and pressing. Some of new methods are laser nano-welding, graphene coating, chemical annealing, plasmonic welding. However, all these methods only solve the problem of electrical conductivity but are helpless for addressing the other two issues. ${ }^{10-17}$

Secondly, pure AgNWs TEs have large area of blank space which is not conductive in AgNWs networks. In fact, AgNWs cover only $20 \%$ of the substrate. However, electrodes must cover all over the active areas for efficient charge extraction/injection because of the typically small lateral conductivity of organic semiconductor for some types of devices like OPVs and OLEDs. So, the concept of composite electrode is put forward. There are many methods to form a composite electrode with a silver nanowire, for example poly(3,4-ethylenedioxythiophene) polystyrene sulfonate (PEDOT:PSS) welding, graphene coating, double-layer structure, electrospun nanofibers, deposition of $\mathrm{MnO}_{2}, \mathrm{TiO}_{2}, \mathrm{ZnO}, \mathrm{AZO}$ particles. The composite electrode can 
easily solve the problem of electrical conductivity and fill the gap between nanowires. Whereas PEDOT:PSS severely limits the device lifetime, and most of the nanoparticles need high temperatures $\left(140{ }^{\circ} \mathrm{C}\right.$ to $\left.250{ }^{\circ} \mathrm{C}\right)$. Zilberberg et al. use $\mathrm{sSnO}_{x} \mathrm{sol}-$ gel process to fabricate the TEs even at room temperature with the method of atomic layer deposition (ALD). ${ }^{23}$ But ALD limits the roll-to-roll production. So, currently, it still cannot achieve a large scale of cost-effective production..$^{10,15-23}$

Thirdly, AgNWs TEs are easily to fall off the substrate due to the poor adhesion, which limits both of its usage and mass production. The composite electrode is very helpful to improve adhesion. However, the current technology is not suitable for roll-to-roll production.

In this work, we report a solution method to fabricate AgNWs TEs on PET substrate at room temperature which is suitable to the roll-to-roll production. More importantly, the fabrication processes are scalable and cost effective. With this method, the $R_{\text {sh }}$ of the TEs can be reduced by four orders while maintaining high transmission. In addition, the gaps between the nanowires are successfully filled and the adhesion between the AgNWs and the substrate are improved. Moreover, this flexible transparent electrode is applicable for any flexible substrate and ultimately for future wearable optoelectronic devices. This will be of great significance to the extension and application of the electrode.

\section{Experiment}

\subsection{Synthesis of AgNWs}

The AgNWs were synthesised by salt-assisted polyol method (Fig. 1a). $30 \mathrm{~mL}$ ethylene glycol (EG) solution of $0.052 \mathrm{~mol} \mathrm{~L}^{-1}$ $\mathrm{AgNO}_{3}$ and $0.067 \mathrm{~mol} \mathrm{~L}^{-1}$ PVP was thoroughly agitated. In addition, another $30 \mathrm{~mL}$ EG solution was prepared and heated at $130{ }^{\circ} \mathrm{C}$ for 1 h. $2.5 \mathrm{~mL}$ EG solution of $6 \times 10^{-4} \mathrm{~mol} \mathrm{~L}^{-1} \mathrm{FeCl}_{3}$ was dropped into the heated $30 \mathrm{~mL}$ EG. After 5-10 min, the mixed solution containing $\mathrm{AgNO}_{3}$ and PVP was added with a dropping speed of $0.5 \mathrm{~mL} \mathrm{~min}^{-1}$ into the heated solution. Finally, post-reaction solution was cooled naturally and then

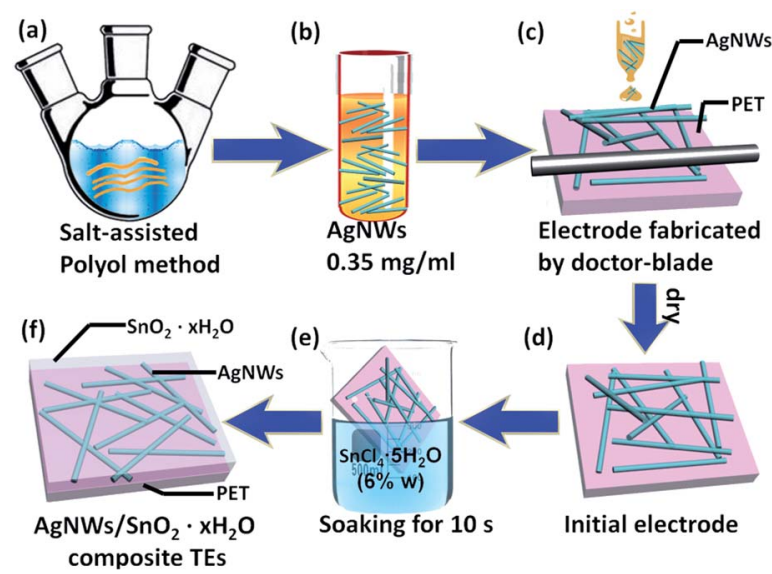

Fig. 1 Flow chart of $\mathrm{AgNWs} / \mathrm{SnO}_{2} \cdot x \mathrm{H}_{2} \mathrm{O}$ composite TEs. (a) Prepare AgNWs by salt-assisted polyol method; (b) disperse AgNWs in ethanol of $0.35 \mathrm{mg} \mathrm{mL}^{-1}$ concentration; (c) Fabricate electrode by doctorblade method; (d) initial electrode; (e) soaking treatment for $10 \mathrm{~s}$; (f) schematic illustration of $\mathrm{AgNWs} / \mathrm{SnO}_{2} \cdot \mathrm{xH}_{2} \mathrm{O}$ composite TEs. filtered by qualified filter paper (intermediate speed). AgNWs were washed out of the filter paper with deionized water. AgNWs were obtained by centrifugation at speed of $2000 \mathrm{rpm}$. Then the precipitation was retained, and the solution was replaced by ethanol. The step of centrifuge separation was repeated for three times.

\subsection{Fabricate of AgNWs TEs}

AgNWs were ultrasonically dispersed in ethanol with the concentration of $0.35 \mathrm{mg} \mathrm{mL}^{-1}$ (Fig. 1b). The AgNWs TEs were fabricated by the doctor-blade on the substrate of PET (Fig. 1c and $\mathrm{d}$ ). And then the films were immersed in aqueous solution of $\mathrm{SnCl}_{4} \cdot 5 \mathrm{H}_{2} \mathrm{O}(6 \% \mathrm{w})$ for $10 \mathrm{~s}$. Finally, the TEs were washed with deionized water and dried.

\section{Results and discussion}

\subsection{Conductivity}

The $R_{\text {sh }}$ of the AgNWs TEs without any post-treatment are as high as $90 \mathrm{k} \Omega \mathrm{sq}^{-1}$. This is a problem that AgNWs TEs usually have, mainly because AgNWs are piled up on the substrates without any contact. Therefore, the improvement of contact is very important for good conductivity. Fortunately, we found a scalable, and cost-effective way to solve this problem. The AgNWs TEs were immersed into the solution of $\mathrm{SnCl}_{4} \cdot 5 \mathrm{H}_{2} \mathrm{O}$ (6\% w) for $10 \mathrm{~s}$. The $R_{\mathrm{sh}}$ of initial AgNWs TEs, $\mathrm{SnO}_{2} \cdot x \mathrm{H}_{2} \mathrm{O}$ film and AgNWs $/ \mathrm{SnO}_{2} \cdot x \mathrm{H}_{2} \mathrm{O}$ composite TEs is shown in Fig. 2. The conductivity of the initial TEs is very poor with the $R_{\text {sh }}$ over 90 $\mathrm{k} \Omega$ (blue pillar). Similarly, the $R_{\mathrm{sh}}$ of the only $\mathrm{SnO}_{2} \cdot x \mathrm{H}_{2} \mathrm{O}$ (red pillar) is $6 \mathrm{k} \Omega$ which is also too high for TEs. Fortunately, the $R_{\mathrm{sh}}$ of the AgNWs $/ \mathrm{SnO}_{2} \cdot x \mathrm{H}_{2} \mathrm{O}$ composite TEs (green pillar) is just 12 $\Omega \mathrm{sq}^{-1}$. The $R_{\mathrm{sh}}$ of AgNWs TEs was reduced dramatically from 90 $\mathrm{k} \Omega \mathrm{sq}^{-1}$ to $12 \Omega \mathrm{sq}^{-1}$ by $99 \%$ or about 4 orders of magnitude.

To found the cause of the increase in conductivity, we analysed the solution. The solution of $\mathrm{SnCl}_{4} \cdot 5 \mathrm{H}_{2} \mathrm{O}$ is not stable and easy to be hydrolysed. The reactions in the aqueous solution of $\mathrm{SnCl}_{4} \cdot 5 \mathrm{H}_{2} \mathrm{O}$ can be expressed as:

$$
\begin{gathered}
\mathrm{SnCl}_{4}+6 \mathrm{H}_{2} \mathrm{O} \rightarrow \mathrm{H}_{2}\left[\mathrm{Sn}(\mathrm{OH})_{6}\right]+4 \mathrm{HCl} \\
\mathrm{SnO}_{2} \cdot 6 \mathrm{H}_{2} \mathrm{O}+6 \mathrm{HCl} \rightarrow \mathrm{H}_{2} \mathrm{SnCl}_{6}+8 \mathrm{H}_{2} \mathrm{O}
\end{gathered}
$$

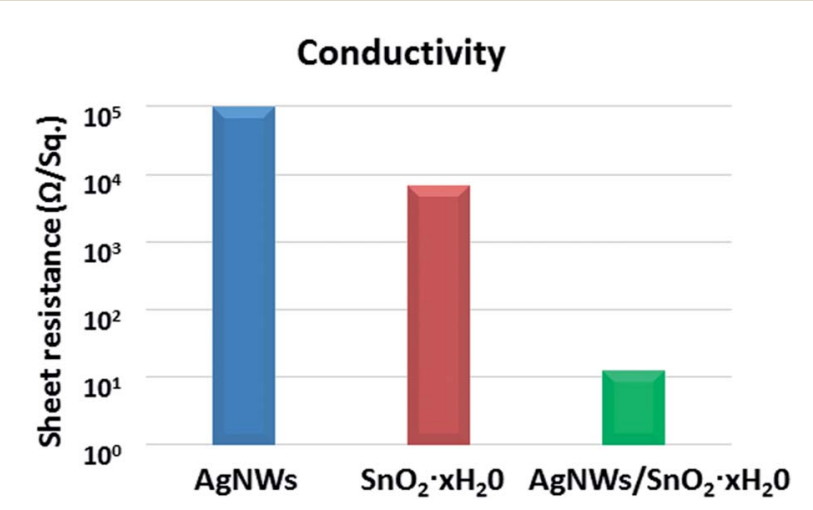

Fig. $2 R_{\text {sh }}$ of initial AgNWs TEs, $\mathrm{SnO}_{2} \cdot x \mathrm{H}_{2} \mathrm{O}$ film and AgNWs/ $\mathrm{SnO}_{2} \cdot \times \mathrm{H}_{2} \mathrm{O}$ composite TEs. 
Table 1 Comparison of electrical properties of composite $\mathrm{TEs}^{25-29}$

\begin{tabular}{lllll}
\hline Composite TEs & Length $(\mu \mathrm{m})$ & Diameter $(\mathrm{nm})$ & $R_{\text {sh }}\left(\Omega \mathrm{sq}^{-1}\right)$ & Transmittance \\
\hline AgNWs/PEDOT:PSS & $20-30$ & $50-100$ & 600 & $90 \%$ \\
AgNWs/PEDOT:PSS & $50-100$ & $50-100$ & 54 & $90 \%$ \\
AgNWs/RGO & $10-20$ & $50-100$ & 20 & $92 \%$ \\
AgNWs/Aa-PDA & $10-20$ & $40-50$ & 5.2 & $94 \%$ \\
AgNWs/sSnO & $10-20$ & $50-100$ & 4.2 & $87 \%$ \\
AgNWs/ZnO & $5-10$ & $50-100$ & 12 & $85 \%$ \\
AgNWs/SnO & $\cdot \mathrm{H}_{2} \mathrm{O}$ & $10-20$ & $50-100$ &
\end{tabular}

where $\mathrm{H}_{2}\left[\mathrm{Sn}(\mathrm{OH})_{6}\right]$ in eqn (1) can be written as $\mathrm{SnO}_{2} \cdot 6 \mathrm{H}_{2} \mathrm{O}$ which will react to $\mathrm{HCl}$ to obtain product $\mathrm{H}_{2} \mathrm{SnCl}_{6}$ that causes the solution to be acidic with $\mathrm{pH}$ value of 2 , as confirmed through experiments. And because the number of $\mathrm{Cl}^{-}$is smaller than $\mathrm{Sn}^{4+}$, there is still some residual of $\mathrm{SnO}_{2} \cdot x \mathrm{H}_{2} \mathrm{O}$. As a result, the hydrolysis of the solution finally obtained the $\mathrm{SnO}_{2}$ colloid and $\mathrm{H}_{2} \mathrm{SnCl}_{6}$.

There are two main effects of $\mathrm{SnCl}_{4} \cdot \mathrm{H}_{2} \mathrm{O}$ solution on TEs, which lead to the $R_{\mathrm{sh}}$ reduction of four orders in magnitude, from $90 \mathrm{k} \Omega \mathrm{sq}^{-1}$ to $12 \Omega \mathrm{sq}^{-1}$.

Firstly, $\mathrm{SnO}_{2} \cdot x \mathrm{H}_{2} \mathrm{O}$ can make the nanowire in close contact and fill the gap of AgNWs TEs. The nanowires will be adsorbed on the substrate due to the strong adhesion of $\mathrm{SnO}_{2}$ with substrate. And adhesion of $\mathrm{SnO}_{2}$ with glass can even reach $20 \mathrm{MPa} . \mathrm{SnO}_{2} \cdot x \mathrm{H}_{2} \mathrm{O}$ can help the nanowires adhere firmly to the surface of the substrate and contact closely to each other for better transmission of electrons. Moreover, the filling of the voids by $\mathrm{SnO}_{2} \cdot x \mathrm{H}_{2} \mathrm{O}$ is very important for electronic devices. The filling of the nonconductive voids can improve the collection of carriers which will improve the performance of electronic devices.

Secondly, $\mathrm{Cl}^{-}$ions could help to bind the AgNWs together and form a weld-like junction which will further increase the conductivity of the film. As discussed in previous studies, ${ }^{21}$ silver atoms in AgNWs may be dissolved slowly in the presence of $\mathrm{Cl}^{-}$and dissolved oxygen in water. Due to redox reactions, it will produce solvated silver ions $\left(\mathrm{Ag}^{+}\right)$. The redox reaction can be written as:

$$
4 \mathrm{Ag}+\mathrm{O}_{2}+2 \mathrm{H}_{2} \mathrm{O} \leftrightarrow 4 \mathrm{Ag}^{+}+4 \mathrm{OH}^{-}
$$

We need to pay attention to the atomic oxygen on the silver surface. It may block the re-deposition of silver ions onto the silver surface. And atomic oxygen on the silver surface comes from molecular oxygen which is adsorbed and dissociated to atomic oxygen between $200 \mathrm{~K}$ and $500 \mathrm{~K}^{24}$ However, concentration of dissolved oxygen in water is very limited so that $\mathrm{Ag}^{+}$ ions could redeposit onto the relatively more active AgNWs surface and junction. The deposition of $\mathrm{Ag}$ at the junction will allow the two nanowires to be successfully welded together and will reduce $R_{\mathrm{c}}$, which is the main source of $R_{\mathrm{sh}}$. So, the $\mathrm{Cl}^{-}$in the solution can help improve the conductivity of TEs.

In Table 1, the electrical properties of different composite TEs are compared and analysed. The last row presents our results, whose perform is seen to be better than other types of electrodes. When the length of the nanowire is doubled, the resistance is reduced by an order of magnitude. In contrast, while the diameter varies at $50-100 \mathrm{~nm}$, the transmittance is maintained at $90 \%$, the longer the nanowires are, the better the electrical properties. And we also found that when the length is the same, the thinner the nanowire is, the better the electrical properties are.

\subsection{Optical properties}

Optical properties are also an important aspect of transparent electrodes. Fig. 3 shows the change of transmittance $(T)$, scattering $(S)$, and haze $(H)$ properties of initial AgNWs TEs and AgNWs $/ \mathrm{SnO}_{2} \cdot x \mathrm{H}_{2} \mathrm{O}$ composite TEs. Haze is a percentage of the transmitted light intensity which is more than 2.5 degrees deviation from the incident light. And haze is equal to the percentage ratio of the scattered light flux $(S)$ within large angles deviating from the incident direction by more than $2.5^{\circ}$ to the transmitted luminous flux (T), i.e.

$$
H=\frac{S}{T} \times 100 \%
$$

where $H$ represents haze, and $S$ represents the large-angle scattered light flux, and $T$ represents transmitted luminous flux. The demand for haze is not the same for different applications. For display devices, haze should be as small as possible

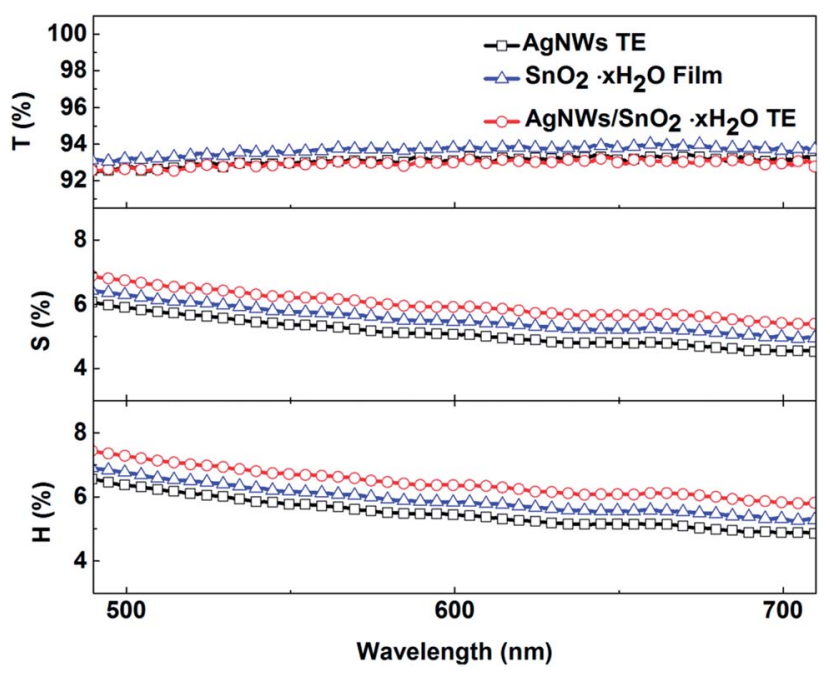

Fig. 3 Transmittance $(T)$, scattering $(S)$, and haze $(H)$ properties of initial AgNWs TEs, $\mathrm{SnO}_{2} \cdot x \mathrm{H}_{2} \mathrm{O}$ film and $\mathrm{AgNWs} / \mathrm{SnO}_{2} \cdot x \mathrm{H}_{2} \mathrm{O}$ composite TES. 
(usually $<1 \%$ ). Yet, for photovoltaic devices, haze can be as large as possible to increase optical path length in devices.

As shown in Fig. 3, for the initial AgNW TEs (black line), fabricated by means of the doctor-blade coating method, the transmittance is over $92.9 \%$ (at $550 \mathrm{~nm}$ wavelength). But the $R_{\mathrm{sh}}$ is too high for the pure AgNW TEs. Similarly, the only $\mathrm{SnO}_{2} \cdot x \mathrm{H}_{2} \mathrm{O}$ film (blue line) has a transmittance of $93 \%$ but its conductivity is not good enough yet. In contrast, for the composite TE, its transmittance is little changed and remained at $92.7 \%$, but its conductivity is significantly improved, also its haze and scattering are increased by about $1 \%$. This means that the presence of the $\mathrm{SnO}_{2} \cdot x \mathrm{H}_{2} \mathrm{O}$ layer has little effect on the transmittance but will enhance the scattering due perhaps to the change of refractive index.

\subsection{Morphology}

Fig. $4 \mathrm{a}$ and $\mathrm{b}$ are the diagram of structure of the initial AgNWs TEs. Fig. 4c and d are the SEM image corresponding to the schematic in Fig. 4a. From Fig. 4c, we can see that the length of AgNWs is about $10-20 \mu \mathrm{m}$, and the diameter is $50-100 \mathrm{~nm}$. As shown in Fig. 4d, AgNWs do not lie flat on the substrate, but tilt arbitrarily. The contact between AgNWs is poor, and some of the nanowires are even turned up. Accordingly, the large resistance of the initial AgNWs TEs probably comes from the poor contact. Therefore, to reduce $R_{\mathrm{sh}}$, we need to first reduce the $R_{\mathrm{c}}$. Fig. $4 \mathrm{~b}$ and e are the schematic and SEM image of the composite TEs, respectively. Fig. 4e shows that $\mathrm{SnO}_{2} \cdot x \mathrm{H}_{2} \mathrm{O}$ filled the gaps between nanowires or even covered the surface of the nanowires. Fig. $4 \mathrm{f}$ is a SEM image of damaged composite TEs which (a)

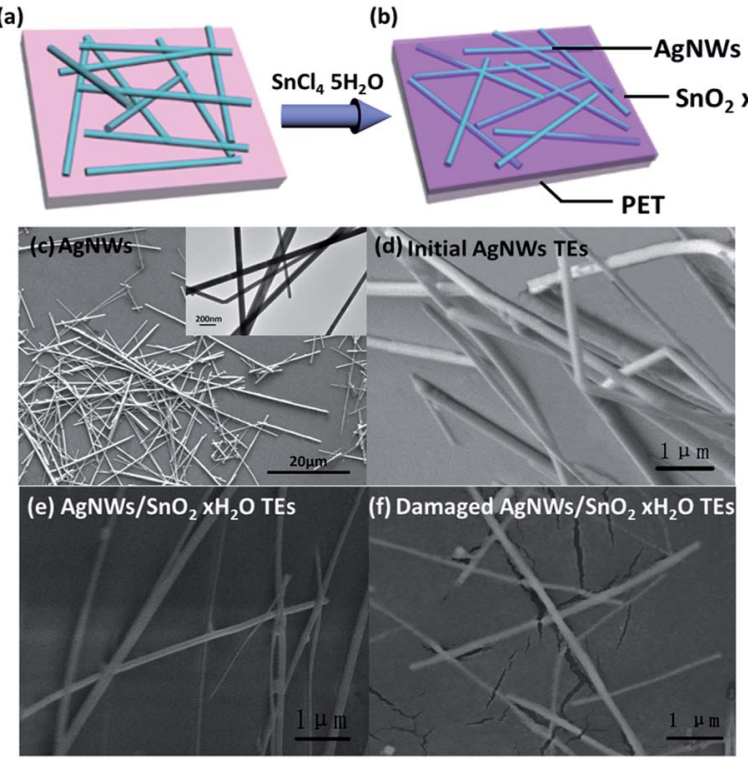

Fig. 4 Diagram of electrode structure and SEM images. (a) Structure of the initial AgNWs TEs; (b) structure of the $\mathrm{AgNWs} / \mathrm{SnO}_{2} \cdot \mathrm{xH}_{2} \mathrm{O}$ composite TEs; (c) AgNWs and the TEM image of AgNWs (top right graph and inset picture); (d) the initial AgNWs TEs as looked from the side; (e) the $\mathrm{AgNWs} / \mathrm{SnO}_{2} \cdot \mathrm{HH}_{2} \mathrm{O}$ composite TEs, (f) the damaged AgNWs $/ \mathrm{SnO}_{2} \cdot x \mathrm{H}_{2} \mathrm{O}$ composite TEs (the composite TE is made on a flexible substrate and then elongated to lead to the tearing of the film).

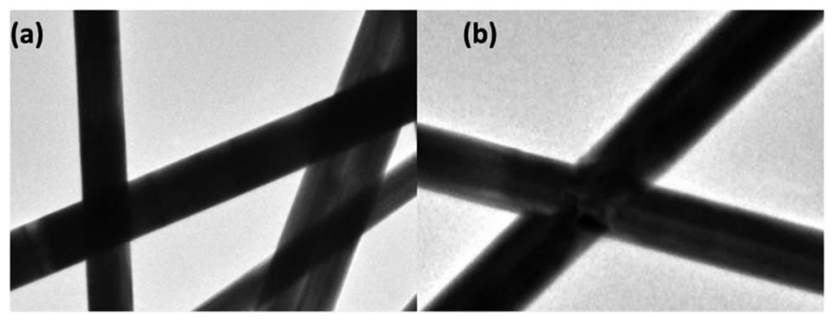

Fig. 5 (a) TEM images of initial AgNWs crossing, (b) TEM images of weld-like AgNWs junction of $\mathrm{AgNWs} / \mathrm{SnO}_{2} \cdot x \mathrm{H}_{2} \mathrm{O}$ composite TEs.

was made on a flexible substrate and pulled to split. As clearly seen through the tearing film in Fig. 4f, the gaps in the nanowire are really filled by the material. Fig. $4 \mathrm{e}$ and $\mathrm{f}$ show that nanowires are all compacted on the substrate and some nanowires are in good contact in $\mathrm{AgNWs} / \mathrm{SnO}_{2} \cdot x \mathrm{H}_{2} \mathrm{O}$ composite TEs.

The junction of the composite TEs is tested by TEM to confirm the contact of the nanowires. Fig. 5a and b show the TEM images of the crossing structure of initial TEs and the composite TEs. For initial AgNWs TEs (Fig. 5a), the connection between the crossed AgNWs is not welded. And the force between the nanowires is mainly van der Waals force. For the $\mathrm{AgNWs} / \mathrm{SnO}_{2} \cdot x \mathrm{H}_{2} \mathrm{O}$ composite TEs, the connection between the crossed AgNWs is welded. The force of the weld-like junction is intermolecular force. Therefore, the nanowires are firmly and stably welded together although exfoliated by the ultrasonic from the substrate. This means that the solution of $\mathrm{SnO}_{2} \cdot x \mathrm{H}_{2} \mathrm{O}$ can help to improve contact of nanowires.

\subsection{Chemical analysis}

The chemical in the nanowire gap is characterized. Fig. 6a shows the TEM-image of the film. Obviously, there is a layer of material, which might be amorphous, deposited onto the surface of the TE and AgNWs. Based on the analysis of the components of the hydrolysis solution, the amorphous substance on the film surface are believed to be $\mathrm{SnO}_{2} \cdot x \mathrm{H}_{2} \mathrm{O}$.

The elemental composition is determined by Energy Dispersive Spectrometer (EDS). Fig. 6c shows the EDS spectrum of the amorphous, and reveals the presence of tin ( $\mathrm{Sn})$, oxygen $(\mathrm{O})$, carbon $(\mathrm{C})$, copper $(\mathrm{Cu})$ and chlorine $(\mathrm{Cl})$ elements in the sample. Carbon is the most abundant. This could be attributed to the amorphous carbon-coated copper grids, which is also the source of copper. The small amount of chlorine comes from the $\mathrm{H}_{2} \mathrm{SnCl}_{6}$ in the solution. The most important thing is verifying the existence of tin and oxygen in the amorphous thought to be $\mathrm{SnO}_{2} \cdot x \mathrm{H}_{2} \mathrm{O}$. To further confirm our speculation, we need to remove the crystalline water from the amorphous to verify the existence of $\mathrm{SnO}_{2}$. When $\mathrm{SnO}_{2} \cdot x \mathrm{H}_{2} \mathrm{O}$ is annealed at $400{ }^{\circ} \mathrm{C}$ for $30 \mathrm{~min}, \mathrm{SnO}_{2} \cdot x \mathrm{H}_{2} \mathrm{O}$ will be turned into the crystallized $\mathrm{SnO}_{2}$. The crystallized $\mathrm{SnO}_{2}$ is characterized by high resolution transmission electron microscopy (HRTEM). The HRTEM image shows a clear lattice spacing of $3.3 \AA$ in Fig. $6 \mathrm{~b}$, which exactly matches to the (110) plane of single crystal $\mathrm{SnO}_{2}$.

Fig. 6d presents the XRD patterns of this crystalline substance. The major XRD peaks at $2 \theta=26.61,33.89,37.95$, 51.78 and $65.94^{\circ}$ are assigned to the (110), (101), (200), (211) and 

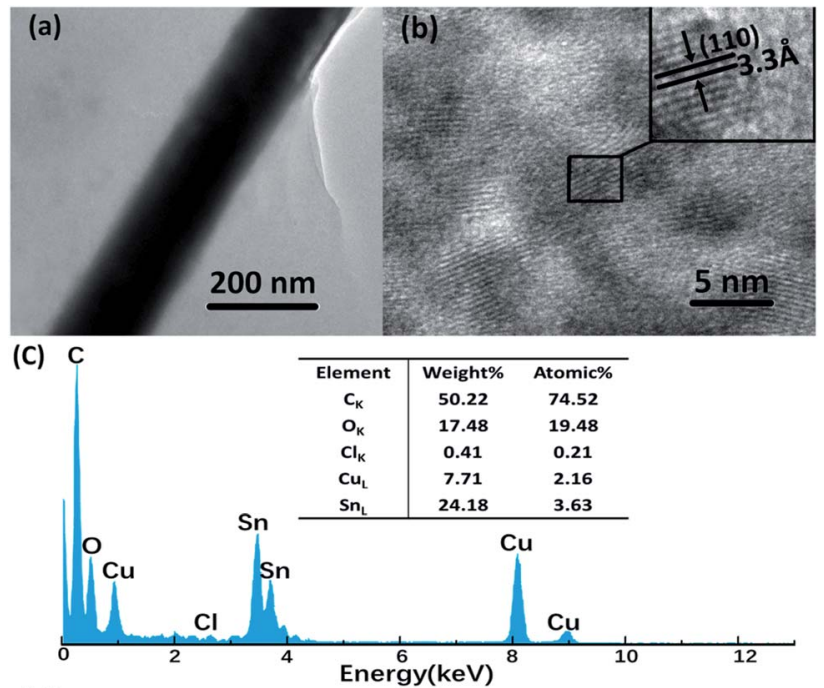

(d)

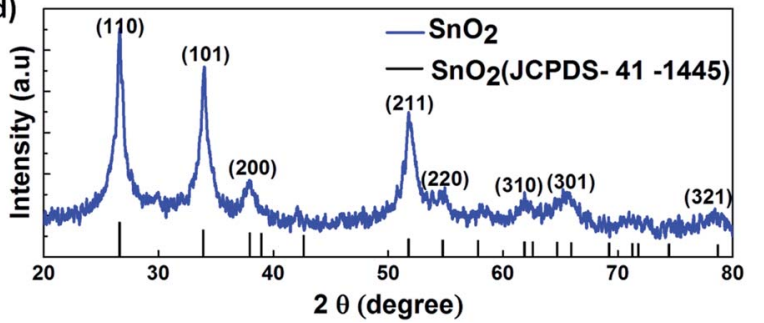

Fig. 6 Microstructure of composite films. (a) TEM images of composite TEs, and (b) HRTEM image of $\mathrm{SnO}_{2}$ annealed at $400{ }^{\circ} \mathrm{C}$ for $30 \mathrm{~min}$. (c) EDS spectrum of $\mathrm{SnO}_{2} \cdot x \mathrm{H}_{2} \mathrm{O}$ along with the table listing the elements and their weight percentage and atomic percentage, (d) XRD patterns of crystallized $\mathrm{SnO}_{2}$ annealed at $400{ }^{\circ} \mathrm{C}$ for $30 \mathrm{~min}$.

(301) reflection planes of tetragonal $\mathrm{SnO}_{2}$, respectively. The diffraction peaks of the products can be well indexed to the known tetragonal rutile $\mathrm{SnO}_{2}$ (JCPDS 41-1445), which confirms the presence of $\mathrm{SnO}_{2}$ in the annealed products. So far, the amorphous material is determined to be $\mathrm{SnO}_{2} \cdot x \mathrm{H}_{2} \mathrm{O}$.

\subsection{Thermal stability}

The thermogravimetric analysis of $\mathrm{SnO}_{2} \cdot x \mathrm{H}_{2} \mathrm{O}$ shows that $\mathrm{SnO}_{2} \cdot x \mathrm{H}_{2} \mathrm{O}$ began to severe loss of water from $100{ }^{\circ} \mathrm{C}$ (Fig. 7a). Nevertheless, crystallization temperature of $\mathrm{SnO}_{2}$ is about $400^{\circ} \mathrm{C}$. If $\mathrm{SnO}_{2} \cdot x \mathrm{H}_{2} \mathrm{O}$ is heated at $100{ }^{\circ} \mathrm{C}$ or $200{ }^{\circ} \mathrm{C}$, we will get the amorphous substance which removed some of the crystal water.

This simple heat treatment surprisingly gives us an unexpected benefit that the adhesion of the film is significantly improved. As we all know, the thin film of AgNWs can easily be erased when we touch it gently due to the poor adhesion of the initial AgNWs TEs. However, there's always a way out! The adhesion of the composite electrodes is improved significantly just with a $100{ }^{\circ} \mathrm{C}$ or $200{ }^{\circ} \mathrm{C}$ heating treatment. This is attributed to the strong adhesive strength between $\mathrm{SnO}_{2}$ and the substrate. When the non-heated composite electrodes were taped and torn down just for one time, the $R_{\mathrm{sh}}$ increased from 12 to $60 \Omega \mathrm{sq}^{-1}$ which is increased by 5 times (black line in Fig. 7b). Nevertheless, the $100{ }^{\circ} \mathrm{C}$ or $200{ }^{\circ} \mathrm{C}$ heating treatment has only a little influence on the conductivity of the composite TEs (Fig. 7b). The $R_{\mathrm{sh}}$ increases only by $3 \Omega \mathrm{sq}^{-1}$. In addition, the thermal (a)
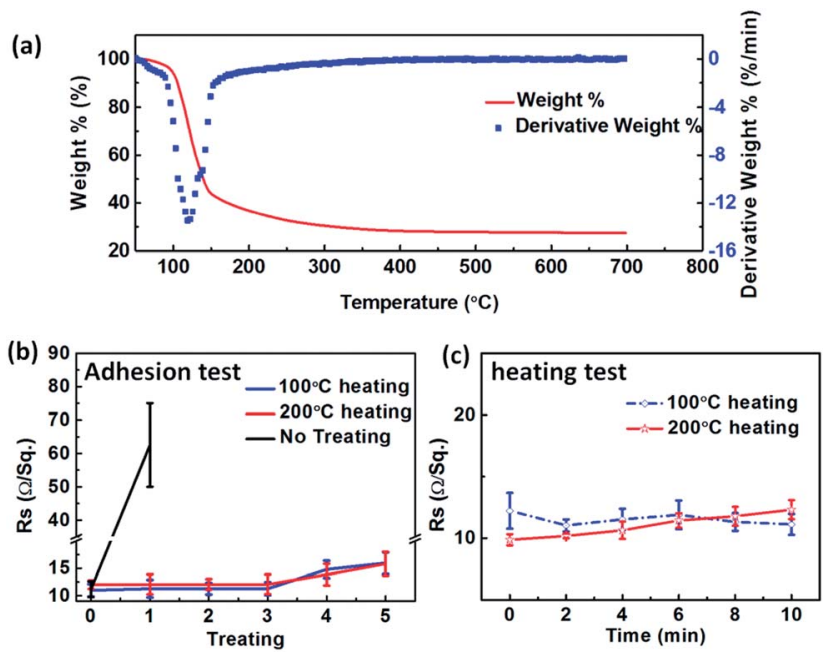

Fig. 7 (a) Thermogravimetric analysis of $\mathrm{SnO}_{2} \cdot x \mathrm{H}_{2} \mathrm{O}$. (b) adhesion test of electrodes. The horizontal axis represents the number of adhesive tape. (c) Resistance change of $\mathrm{AgNWs} / \mathrm{SnO}_{2} \cdot \mathrm{xH}_{2} \mathrm{O}$ composite electrodes during heating.

stability of the heated composite electrode is quite good. The $R_{\mathrm{sh}}$ of the film changes little but maintains at about $12 \Omega \mathrm{sq}^{-1}$ during heating (Fig. 7c). In conclusion, the adhesion of the composite TEs can be improved by heat treatment, and the TEs can also tolerate adhesive tape test.

XPS spectra are used to characterize the initial composite TEs and the heated composite TEs at $200^{\circ} \mathrm{C}$. We want to know whether the heat treatment has other effects on the electrode, in addition to the loss of crystalline water. Fig. 8 shows the X-ray photoelectron spectroscopy (XPS) of the composite TEs. The XPS survey spectrum (Fig. 8a) shows the peaks of Ag 3d doublet, Sn 3d doublet, Sn 4d and $\mathrm{O}$ 1s peaks. There are two peaks with binding energies of $368.3 \mathrm{eV}$ for $\mathrm{Ag} 3 \mathrm{~d}_{5 / 2}$ and $374.3 \mathrm{eV}$ for $\mathrm{Ag} 3 \mathrm{~d}_{3 / 2}$ in the regional spectrum (Fig. 8b) of $\mathrm{Ag} 3 \mathrm{~d}$. This is consistent with the standard binding energy of pure silver. The peaks at a binding energy of 486.6, 495.3 and $27.1 \mathrm{eV}$ (Fig. 8c) can agree with the $\mathrm{Sn} 3 \mathrm{~d}_{5 / 2}, \mathrm{Sn}$
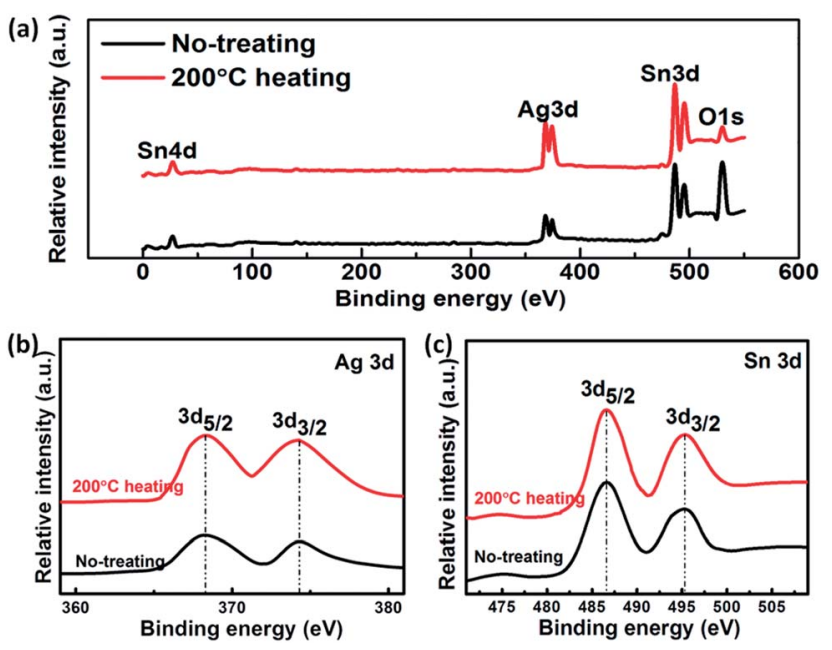

Fig. 8 XPS spectra of $\mathrm{SnO}_{2} \cdot x \mathrm{H}_{2} \mathrm{O}$. (a) General XPS spectra of initial and $200{ }^{\circ} \mathrm{C}$ heating $\mathrm{SnO}_{2} \cdot x \mathrm{H}_{2} \mathrm{O}$. (b) Ag $3 \mathrm{~d} X P S$ spectrum of $\mathrm{SnO}_{2} \cdot x \mathrm{H}_{2} \mathrm{O}$. (c) Sn 3d XPS spectrum of $\mathrm{SnO}_{2} \cdot \times \mathrm{H}_{2} \mathrm{O}$. 
$3 \mathrm{~d}_{3 / 2}$ and $\mathrm{Sn} 4 \mathrm{~d}_{3 / 2}$, respectively. Their standard combination can be very close to the previously reported results, indicating that the valence states of $\mathrm{Ag}$ and $\mathrm{Sn}$ are 0 and +4 , respectively. This means that the heat treatment at $200{ }^{\circ} \mathrm{C}$ does not cause a change in the valence of the composite electrode.

\section{Conclusions}

As reported previously, AgNWs transparent electrode usually has the problems of poor initial conductivity and intrinsic gap between nanowires. This paper demonstrated a simple solutionprocessed method for a scalable, cost effective and flexible AgNWs TEs. Compared with others, this method is characterized by the gap of the silver nanowire network filled by $\mathrm{SnO}_{2} \cdot x \mathrm{H}_{2} \mathrm{O}$. The electrode forms a new kind of $\mathrm{AgNWs} / \mathrm{SnO}_{2} \cdot \mathrm{xH}_{2} \mathrm{O}$ composite structure just with $10 \mathrm{~s}$ immersion in solution of $\mathrm{SnCl}_{4} \cdot 5 \mathrm{H}_{2} \mathrm{O}$ at room temperature. It is confirmed that aqueous solution of $\mathrm{SnCl}_{4} \cdot 5 \mathrm{H}_{2} \mathrm{O}$ could help to reduce the $R_{\mathrm{sh}}$ by four orders of magnitude, from $90 \mathrm{k} \Omega \mathrm{sq}^{-1}$ to $12 \Omega \mathrm{sq}^{-1}$, while keep transmittance of about $92 \%$ at $550 \mathrm{~nm}$. In addition, the adhesive forces between the AgNWs and the substrate is improved. The optimization of the performance is attributed to the effects of $\mathrm{SnCl}_{4} \cdot 5 \mathrm{H}_{2} \mathrm{O}$ solution which mainly contains $\mathrm{Cl}^{-}$and $\mathrm{SnO}_{2} \cdot x \mathrm{H}_{2} \mathrm{O}$ because of hydrolysis. There are two main effects on AgNWs TEs. Firstly, $\mathrm{Cl}^{-}$can help to fuse the contact of nanowires which reduces $R_{\mathrm{c}}$ of TEs. Secondly, $\mathrm{SnO}_{2} \cdot x \mathrm{H}_{2} \mathrm{O}$ can help to fill the space of networks. The conductivity of the composite TEs is improved attributed to this important filling. At the same time, the coverage on the active areas can improve the efficiency of charge extraction/injection for devices. Surprisingly, the adhesion of AgNWs TEs can be significantly improved with 100 or $200{ }^{\circ} \mathrm{C}$ heating. What's more, the simple solution-method at room temperature makes it possible for the roll-to-roll production.

\section{Conflicts of interest}

There are no conflicts to declare.

\section{Acknowledgements}

This work is partially funded by the Natural Science Foundation of Hubei Province (2016CFB266), and State Key Laboratory of Materials Processing and Die \& Mould Technology, Huazhong University of Science and Technology (P2018-012), and Science and technology research project of Hubei Provincial Education Department (B2016442), Doctoral independent research talent fund (2015CYBSKY005) and Key Fund of College (2017CYZDKY001).

\section{Notes and references}

1 E. Chen, H. Xie, J. Huang, H. Miu, G. Shao, Y. Li, T. Guo, S. Xu and Y. Ye, Opt. Express, 2018, 26, 3466.

2 S. W. Apayoon, C. W. Park, S. J. Kim, N. S. Roh, S. I. Kim, W. J. Lee, et al., J. Chromatogr. A, 2017, 625, 56.
3 K. Lee, J. W. Shin, J. H. Park, J. Lee, C. W. Joo and J. I. Lee, ACS Appl. Mater. Interfaces, 2016, 8, 17409.

4 Y. Park, L. Bormann, L. Müller-Meskamp, K. Vandewal and K. Leo, Org. Electron., 2016, 36, 68.

5 S. Hou, J. F. Chen, M. Song, Y. Zhu, Y. J. Jan, S. H. Chen, et al., ACS Nano, 2017, 11, 8167.

6 G. S. Liou, C. Y. Chou and H. S. Liu, RSC Adv., 2016, 6, 6138661392.

7 S. L. Ji, W. W. He and K. Wang, Small, 2014, 10, 4951.

$8 \mathrm{~K}$. Ghaffarzadeh and R. Das, Transparent Conductive Films (TCF) 2014-2024: Forecasts, Markets, Technologies, IDTechEx, 2014.

9 J. Y. Kim, E. J. Lee, Y. H. Kim, D. K. Hwang and W. K. Choi, RSC Adv., 2016, 6, 11702.

10 J. Y. Kim, Y. S. Kim, E. J. Lee, J. T. Lee, D. K. Hwang and W. K. Choi, RSC Adv., 2016, 6, 64428-64433.

11 M. Lomascolo, G. Colangelo and M. Milanese, Renewable Sustainable Energy Rev., 2015, 43, 1182.

12 M. Arango, A. Cokeley and J. Beard, Thin Solid Films, 2015, 596, 167.

13 J. Ha, B. J. Lee, D. J. Hwang and D. Kim, RSC Adv., 2016, 6, 86232.

14 B. Hwang, M. Park, T. Kim and S. M. Han, RSC Adv., 2016, 6, 4770.

15 S. J. Lee, Y. H. Kim, J. K. Kim, H. Baik, J. H. Park and J. Lee, Nanoscale, 2014, 6, 11828.

16 E. C. Garnett, W. Cai, J. J. Cha, F. Mahmood, S. T. Connor and M. G. Christoforo, Nat. Mater., 2012, 11, 241.

17 S. H. Park, C. M. Hong and H. L. Dong, RSC Adv., 2016, 6, 50158.

18 R. Song, X. Li, F. Gu, L. Fei, Q. Ma and Y. Chai, RSC Adv., 2016, 6, 91641.

19 Z. Qiao, X. Yang, S. Yang, L. Zhang and B. Cao, Chem. Commun., 2016, 52, 7008.

20 R. Zhu, C. H. Chung, K. C. Cha, W. Yang, Y. B. Zheng and H. Zhou, ACS Nano, 2011, 5, 9877.

21 J. Han, S. Yuan, L. Liu, X. Qiu, H. Gong, X. Yang, C. Li, Y. Hao and B. Cao, J. Mater. Chem. A, 2015, 3, 5375.

22 T. Stubhan, J. Krantz, N. Li, F. Guo, I. Litzov and M. Steidl, Sol. Energy Mater. Sol. Cells, 2012, 107, 248.

23 K. Zilberberg, F. Gasse, R. Pagui, A. Polywka, A. Behrendt and S. Trost, Adv. Funct. Mater., 2014, 24, 1671.

24 F. B. Mongeot, A. Cupolillo and U. Valbusa, Chem. Phys. Lett., 1997, 270, 345.

25 J. Lee, P. Lee, H. B. Lee, et al., Adv. Funct. Mater., 2014, 23, 4171-4176.

26 I. K. Moon, J. I. Kim, H. Lee, K. Hur, W. C. Kim and H. Lee, Sci. Rep., 2013, 3, 1112.

27 Y. Jin, D. Deng, Y. Cheng, L. Kong and F. Xiao, Nanoscale, 2014, 6, 4812.

28 T. Kim, A. Canlier, C. Cho, V. Rozyyev, J. Y. Lee and S. M. Han, ACS Appl. Mater. Interfaces, 2014, 6, 13527.

29 F. S. F. Morgenstern, D. Kabra, S. Massip, T. J. K. Brenner, P. E. Lyons and J. N. Coleman, Appl. Phys. Lett., 2011, 99, 183307. 